\title{
ORIGINAL
}

\section{CADENA DEL FRÍO PARA LA CONSERVACIÓN DE LAS VACUNAS EN LOS CENTROS DE ATENCIÓN PRIMARIA DE UN AREA DE MADRID: MANTENIMIENTO Y NIVEL DE CONOCIMIENTOS}

\author{
Paloma Ortega Molina, Paloma Astasio Arbiza, Romana Albaladejo Vicente, M. ${ }^{a}$ Luisa Gómez \\ Rábago, José Ramón de Juanes Pardo y Vicente Domínguez Rojas
}

Departamento de Medicina Preventiva y Salud Pública. Facultad de Medicina. Universidad Complutense de Madrid.

\section{RESUMEN}

Fundamento. Las vacunas son medicamentos termolábiles y para garantizar su inmunogenicidad y eficacia protectora, dentro de los programas de inmunización, es imprescindible mantener la cadena de frío. El elemento fundamental en esta cadena es el personal responsable de las vacunas, que debe conocer las características de estabilidad de cada preparado con el fin de evitar errores durante su manipulación. El objetivo de este trabajo fue conocer cómo se realiza el mantenimiento de la cadena del frío en equipos de atención primaria de un área sanitaria de la Comunidad Autónoma de Madrid, así como establecer el grado de información que poseen los responsables de las vacunas con respecto a la termoestabilidad de las mismas.

Métodos. Se ha realizado un estudio transversal en 46 puntos de vacunación en atención primaria. La recogida de los datos se realizó mediante entrevista personal por un único investigador.

Resultados. La tasa de participación fue del 93,5\% (43/46). En todos los casos existía termómetro de máxima y mínima y registro mensual de la temperatura. Se observó una temperatura inadecuada en tres ocasiones $(6,97 \%)$. El porcentaje de profesionales que conocía el efecto que la congelación producía sobre las vacunas fue muy diverso: $53.5 \%, 51.2 \%, 44.2 \%$ y $53.5 \%$ para difteria-tétanos-pertussis (DTP), hepatitis B (VHB), polio oral (VPO) y rubéola-sarampión-paperas (RSP) respectivamente. Y sólo el 32\% conocía el test de agitación.

Conclusión. La formación de los profesionales sobre el efecto que las altas temperaturas ocasionan en las vacunas era correcta, pero es necesario reforzar su formación sobre la inestabilidad que presentan los preparados adsorbidos cuando se someten a congelación.

Palabras Clave: Vacunas. Atención primaria de salud. Personal sanitario. Control de calidad. Cadena del frío.

Correspondencia:

Paloma Ortega Molina

Departamento de Medicina Preventiva y Salud Pública

Facultad de Medicina. Pabellón 2, 2. ${ }^{\mathrm{a}}$ planta

Avda/ Complutense s/n

28040- Madrid

Correo electrónico: pomolina@med.ucm.es

\section{ABSTRACT}

\section{Vaccine Storage Cold Chain at Primary Care Centers in one Area of Madrid: Keeping the Chain Intact and Degree of Knowledge}

Background: Vaccines are heat-labile medications, and to guarantee their immunogenicity and safeguarding effectiveness as part of immunization programs, it is absolutely essential that the «Cold Chain» go unbroken. Fundamental thereto is the personnel responsible for the vaccines, who must know the stability-related characteristics of each preparation so as to prevent handling errors. The purpose of this study was that of ascertaining how the cold chain is kept intact in primary care systems in one healthcare area of the Autonomous Community of Madrid, as well as determining the degree of information possessed by those responsible for vaccines as far as their heat-stability is concerned.

Methods. A cross-sectional study has been made at 46 primary care vaccination points. The data was gathered by means of a personal interview by one single researcher.

Results. The participation rate was $93.5 \%$ (43/46). In all cases, there was a maximum and minimum thermometer and monthly temperature record. An unsuitable temperature was found in three cases $(6.97 \%)$. The percentage of professionals who were aware of the effect freezing has on vaccines varied greatly: $53.5 \%, 51.2 \%, 44.2 \%$ and $53.5 \%$ for diphtheria-tetanus-pertussis (DTP), hepatitis B (HBV), oral polio (OPV) and measles-mumps-rubella (MMR) respectively. And only 32\% were familiar with the shake test.

Conclusion. The professionals were found to be properly trained regarding the effect which high temperatures have on vaccines, but it is necessary for their training with regard to the instability of adsorbed preparations when frozen must be further strengthened.

Keywords: Vaccines. Primary health care. Healthcare professionals. Cold Chain. Quality control. 


\section{INTRODUCCIÓN}

En nuestro país los programas de inmunización se desarrollan en el ámbito de la atención primaria de salud, puesto que es el punto inicial de contacto entre el ciudadano y el sistema sanitario. Esto reporta beneficios importantes tanto en accesibilidad como en cobertura, constituyendo la vacunación una actividad esencial de los equipos de atención primaria.

Actualmente disponemos de un amplio número de vacunas que son seguras y efectivas, propiedades que, sin embargo, no son suficientes para garantizar la eficiencia de los programas de vacunación. Es imprescindible que dichas vacunas sean accesibles a la población diana y además que lleguen en perfecto estado de conservación, de forma que se podría garantizar tanto su inmunogenicidad como su eficacia protectora ${ }^{1,2}$.

La estabilidad de las vacunas puede verse afectada por múltiples factores (luz, temperatura, humedad, cepa vacunal) que pueden ocasionar la pérdida de capacidad inmunizante, de forma acumulativa e irreversible, incrementándose con el tiempo de exposición a dichos factores ${ }^{3-5}$.

El carácter termosensible de las vacunas, que hace necesario su conservación entre $2-8^{\circ} \mathrm{C}$, puede comprometer su efectividad si se producen errores durante su transporte, almacenamiento y manipulación, siendo por tanto imprescindible una correcta planificación logística de los programas de inmunización, así como el adecuado mantenimiento de la cadena del frío durante todo el proceso. En este sentido, la bibliografía recoge distintos estudios en los que se responsabiliza a la inadecuada conservación y manipulación de las vacunas como la posible causa de casos de sarampión en personas inmunizadas en Canadá6, ${ }^{6,}$ Chen argumentaba que la existencia de deficiencias en el mantenimiento de la cadena del frío puede ser considerada como una de las cinco posibles causas del brote de difteria en la antigua Unión
Soviética en $1990^{8}$; y más recientemente Gold en Australia detectó un incremento considerable de los costes de una campaña de vacunación de adultos frente a tétanos y difteria, debido a la congelación de los preparados por un incorrecto almacenamiento de los mismos 9 .

Si bien la estabilidad de las vacunas atenuadas se ve comprometida fundamentalmente por su exposición a temperaturas superiores a $8^{\circ} \mathrm{C}$, las inferiores a $0^{\circ} \mathrm{C}$ pueden inactivar por congelación distintas vacunas, en general todas las que llevan adyuvan$\mathrm{te}^{4,8,14}$. En este tipo de vacunas adsorbidas es preciso observar siempre su aspecto externo y realizar la prueba de agitación antes de su administración, con el fin de comprobar si ha floculado, quedando en este caso la vacuna inactivada ${ }^{4,15}$. Especial cuidado requiere la vacuna frente al virus de hepatitis B cuyo punto de congelación se sitúa en los $-0,5^{\circ} \mathrm{C}^{15}$, siendo por el contrario una de las vacunas más estables a altas temperaturas, lo que según Otto ${ }^{12}$ permitirá en un futuro próximo que su almacenamiento y transporte pueda realizarse sin necesidad de la cadena del frío.

El único método que en la actualidad nos permite garantizar la inmunogenicidad y eficacia protectora de una vacuna desde su elaboración hasta su administración, es el mantenimiento de la cadena del frío. Para que ésta sea operativa es necesario que los profesionales implicados en sus distintas fases, almacenamiento, transporte y administración, tengan una formación adecuada sobre la termolabilidad de los productos que manipulan.

Si bien es cierto que el mantenimiento de la cadena del frío cuenta, a priori, en nuestro ámbito con todos los medios necesarios y suficientes para garantizar la perfecta manipulación de las vacunas, no es anecdótico, pero si preocupante, ver reflejados en la literatura distintos estudios realizados recientemente en países industrializados ${ }^{6,9,16-20}$, que evidencian serios problemas durante el al- 
macenamiento y manipulación de las mismas en los puntos de vacunación, así como serias deficiencias en el nivel de formación del personal responsable de la cadena del frío $^{21,} 22$. En este sentido, ya Bishai en $1992^{17}$, en un estudio realizado en California, mencionaba que el $36 \%$ de los responsables de vacunas en los puntos de administración desconocían que la congelación podía inactivar determinados preparados.

Ante estos hechos, nos preguntamos qué sucede en nuestro medio. Para responder a este interrogante realizamos el presente estudio con el objetivo de conocer cómo se realiza el mantenimiento de la cadena del frío en Equipos de Atención Primaria (EAP) de un área sanitaria de la Comunidad Autónoma de Madrid, así como valorar el grado de información del responsable de vacunas con respecto a la termolabilidad de las mismas.

\section{MATERIAL Y MÉTODOS}

Durante el periodo de tiempo comprendido entre mayo y junio de 2000 se ha llevado a cabo un estudio transversal en los 46 puntos de vacunación de atención primaria de salud, pertenecientes a un área sanitaria de Madrid. Previamente a su inicio se obtuvo la autorización pertinente de la Dirección para su realización.

Dicha área sanitaria se encuentra ubicada geográficamente en la zona sureste de la Comunidad de Madrid, estando distribuida en cuatro distritos, tres urbanos y uno de estructura mixta urbano-rural, y proporciona asistencia sanitaria a 615.287 habitantes, de los cuales 88.352 eran menores de 14 años.

Para la recogida de la información se diseñó un cuestionario que constaba de 30 items (anexo 1), dividido en dos partes; la primera nos permitiría recoger los datos referentes al mantenimiento de la cadena del frío en los puntos de vacunación y la segun- da nos reflejaría el grado de información del responsable de vacunas sobre la termoestabilidad de las mismas, así como el nivel de conocimiento sobre las normas de actuación recomendadas por la Consejería de Salud de la Comunidad de Madrid ante cualquier problema durante su conservación ${ }^{23}$. Dicho cuestionario había sido validado en un estudio piloto efectuado con anterioridad en otros centros de vacunación.

La recogida de los datos se realizó mediante la visualización del interior del frigorífico y a través de entrevista personal estandarizada. Con el fin de minimizar el sesgo de mala clasificación, hemos de puntualizar que tanto la entrevista como la inspección del frigorífico, fue realizada en todos los casos por el mismo investigador, garantizándose en todo momento la confidencialidad de los datos. Así mismo, la recogida de la información en todos los puntos de vacunación se llevó a cabo en un período de 35 días, no existiendo por tanto variaciones ni estacionales ni asistenciales, que pudieran interferir en los resultados obtenidos ${ }^{24}$.

Durante la inspección visual del frigorífico se registraron los siguientes datos: temperatura que marcaba el termómetro de máximas y mínimas, ubicación de los viales de vacuna en su interior y/o en la puerta del mismo, presencia de viales caducados y/o abiertos, existencia de acumuladores de frío y/o botellas de suero salino para garantizar la temperatura óptima del frigorífico y presencia/ausencia de alimentos o bebidas. Así mismo se recababa información específica sobre la existencia de averías en el frigorífico o cortes en el suministro eléctrico en el punto de vacunación en los últimos doce meses.

El análisis de los datos se realizó mediante el programa SPSS $10.0^{25}$, que nos permitió el cálculo y comparación de las frecuencias y medias de las distintas variables entre zonas urbanas y rurales. Se consideró como nivel de significación aquellos valores de $\mathrm{p}$ $<0,05$. 


\section{RESULTADOS}

De los 46 responsables de vacunas contactados, 43 participaron en el estudio, obteniendo una tasa de participación del 93,5\%; de ellos $25(58,14 \%)$ desarrollaban su actividad asistencial en zonas urbanas y 18 $(41,86 \%)$ en zonas rurales.

La media de edad de los participantes fue de 41,56 $\pm 8,85$ años, con una edad mínima de 24 años y una máxima de 60 años, no existiendo diferencias entre zonas urbanas y rurales. El 72,1\% de la población pertenecía al sexo femenino, porcentaje que se incrementa al $92 \%$ en los centros urbanos y desciende al 44,4\% en los rurales. El 97,7\% de los profesionales responsables de la cadena del frío era personal de enfermería, existiendo en el $93 \%$ de los puntos de vacunación (40/43) una única persona responsable.

Como se ha mencionado en el apartado de material y métodos, el cuestionario recogía información sobre el mantenimiento de la cadena del frío en los puntos de vacunación, quedando expresados en la tabla 1 los datos más relevantes.

Tabla 1

Características del frigorífico utilizado para el almacenamiento de las vacunas en los 43 puntos de vacunación estudiados

\begin{tabular}{|c|c|c|c|}
\hline \multirow[b]{2}{*}{ Variable } & \multicolumn{3}{|c|}{ Puntos de Vacunación } \\
\hline & $\begin{array}{c}\text { Total } \\
\left(N .^{\circ}=43\right)\end{array}$ & $\begin{array}{l}\text { Urbanos } \\
\left(N . .^{\circ}=25\right)\end{array}$ & $\begin{array}{l}\text { Rurales } \\
\left(N .{ }^{\circ}=18\right)\end{array}$ \\
\hline \multicolumn{4}{|l|}{ Tipo de Frigorífico } \\
\hline Doméstico & $27(62,8 \%)$ & $11(44 \%)$ & $16(88,9 \%)$ \\
\hline Sanitario & $16(37,2 \%)$ & $14(56 \%)$ & $2(11,1 \%)$ \\
\hline \multicolumn{4}{|l|}{ Utilización del Frigorífico } \\
\hline Exclusivo vacunas & $22(51,2 \%)$ & $15(60 \%)$ & $7(38,9 \%)$ \\
\hline Todo medicamento termosensible & $21(48,8 \%)$ & $10(40 \%)$ & $11(61,1 \%)$ \\
\hline \multicolumn{4}{|l|}{ Conexión a la red eléctrica } \\
\hline Directamente & $41(95,5 \%)$ & $24(96 \%)$ & $17(94,4 \%)$ \\
\hline Derivaciones & $2(4,7 \%)$ & $1(4 \%)$ & $1(5,6 \%)$ \\
\hline \multicolumn{4}{|l|}{ Conexión a red eléctrica de emergencia } \\
\hline Sí & $1(2,3 \%)$ & $1(4 \%)$ & - \\
\hline No & $42(97,7 \%)$ & $24(96 \%)$ & $18(100 \%)$ \\
\hline \multicolumn{4}{|c|}{ Necesidad apertura de frigorífico para leer $\mathrm{T}^{\mathrm{a}}$} \\
\hline Sí & $40(93 \%)$ & $23(92 \%)$ & $17(94,4 \%)$ \\
\hline No & $3(7 \%)$ & $2(8 \%)$ & $1(5,6 \%)$ \\
\hline \multicolumn{4}{|l|}{ Dispone de alarma en la puerta } \\
\hline Sí & $2(4,7 \%)$ & $1(4 \%)$ & $1(5,6 \%)$ \\
\hline No & $41(95,3 \%)$ & $24(96 \%)$ & $17(94,4 \%)$ \\
\hline \multicolumn{4}{|l|}{ Averías últimos 12 meses } \\
\hline No & $14(32,6 \%)$ & $7(28 \%)$ & $7(38,9 \%)$ \\
\hline 1 avería & $22(51,2 \%)$ & $16(64 \%)$ & $6(33,3 \%)$ \\
\hline 2 averías & $6(14,0 \%)$ & $2(8 \%)$ & $4(22,2 \%)$ \\
\hline No sabe & $1(5,6 \%)$ & - & $1(5,6 \%)$ \\
\hline \multicolumn{4}{|l|}{ Vacunas situadas en la puerta } \\
\hline Sí & $6(14 \%)$ & $2(8 \%)$ & $4(22,2 \%)$ \\
\hline No & $37(86 \%)$ & $23(92 \%)$ & $14(77,8 \%)$ \\
\hline
\end{tabular}

El $100 \%$ de los frigoríficos disponían en su interior de termómetro de máxima y mínima, para cuya lectura era imprescindible abrir la nevera en el $93 \%$ de los casos. En el $100 \%$ de las ocasiones existía un registro gráfico mensual de las temperaturas máxi- 
mas y mínimas alcanzadas por el frigorífico, si bien es cierto que sólo en el 76,7\% de los casos se realizaban dos lecturas diarias (inicio y final de la jornada laboral). En el momento de la visita se registró la temperatura que marcaba dicho termómetro y sólo en el $6,97 \%$ (3/43) comprobamos como ésta no se encontraba dentro del rango óptimo, en dos ocasiones la temperatura era de $-1^{\circ} \mathrm{C}$ y en otra $+8,5^{\circ} \mathrm{C}$.

En la totalidad de los centros visitados se procedía correctamente en cuanto al mantenimiento de la temperatura en el interior del frigorífico ya que en todos los casos se observó la presencia de acumuladores de frío y/o botellas de suero, y en aquellos que requerían ser descongelados periódicamente se adoptaban las medidas que garantizaban el mantenimiento de la cadena del frío durante el proceso. Así mismo, el 100\% de los responsables estaban perfectamente informados de las normas de actuación ante cualquier problema surgido durante el almacenamiento de las vacunas.

Los viales en la rutina diaria eran extraídos del frigorífico a demanda en el $90,7 \%$ de los centros (39/43), y en el 4,7\% (2/43) eran transportados y mantenidos hasta el momento de su administración en bateas con acumuladores de frío.

Hemos de resaltar que en 6 frigoríficos (4 rurales y 2 urbanos) se observó la presencia de viales situados en la puerta de la ne- vera, sin embargo en ningún caso se constató la presencia de vacunas caducadas ni de alimentos y/o bebidas, y en dos puntos de vacunación rurales se encontraba en uso un vial multidosis de vacuna antipoliomielítica oral que era conservado adecuadamente.

La tabla 2 refleja el grado de información que el personal implicado en la cadena del frío poseía sobre la termoestabilidad de las vacunas. La totalidad de los encuestados conocía de forma global la posible pérdida de actividad de las vacunas debido a su exposición tanto a altas como a bajas temperaturas. Ahora bien, cuando se les interrogó sobre el efecto que la congelación podía generar sobre cuatro preparados que se administran sistemáticamente a la población infantil (DTP, VHB, VPO, RSP), mediante cuatro cuestiones independientes, nos encontramos que el porcentaje de profesionales que respondieron adecuadamente a cada una de ellas fue: $53,5 \%, 51,2 \%, 44,2 \%$ y $53,5 \%$ respectivamente (tabla 2 ).

El cuestionario incluía una pregunta que reflejaba el conocimiento del responsable de vacunas sobre el test de agitación para la detección de vacunas floculadas como consecuencia de su congelación, que nos puso de manifiesto que sólo $14(32,6 \%)$ lo conocían. El $48 \%$ de los profesionales de los centros urbanos conocían dicho test, mientras que sólo lo conocía el $11,1 \%$ de los responsables de centros rurales, siendo esta diferencia estadísticamente significativa (tabla 2). 
Tabla 2

Variables que reflejan el grado de información con respecto a la termoestabilidad de las vacunas ${ }^{*} \mathbf{p}<\mathbf{0 , 0 5}$

\begin{tabular}{|c|c|c|c|}
\hline \multirow{2}{*}{ Variable } & \multicolumn{3}{|c|}{ Puntos de Vacunación } \\
\hline & $\begin{array}{c}\text { Total } \\
\left(N .^{o}=43\right)\end{array}$ & $\begin{array}{l}\text { Urbanos } \\
\left(N .{ }^{\circ}=25\right)\end{array}$ & $\begin{array}{c}\text { Rurales } \\
\left(N .{ }^{\circ}=18\right)\end{array}$ \\
\hline \multicolumn{4}{|c|}{ Rango óptimo conservación } \\
\hline $2-8 .{ }^{\circ} \mathrm{C}$ & $38(88,4 \%)$ & $21(84 \%)$ & $17(94,4 \%)$ \\
\hline $0-8 .{ }^{\circ} \mathrm{C}$ & $2(4,7 \%)$ & $1(4 \%)$ & $1(5,6 \%)$ \\
\hline $2-10 .^{\circ} \mathrm{C}$ & $3(7,0 \%)$ & $3(12 \%)$ & - \\
\hline \multicolumn{4}{|c|}{ Medida de la Temperatura diaria } \\
\hline 2 veces/día & $33(76,7 \%)$ & $21(84 \%)$ & $12(66,7 \%)$ \\
\hline 1 vez/día & $9(20,9 \%)$ & $4(16 \%)$ & $5(27,8 \%)$ \\
\hline $1-2$ veces/semana & $1(2,3 \%)$ & - & $1(5,6 \%)$ \\
\hline \multicolumn{4}{|l|}{ Test Agitación } \\
\hline Conoce & $14(32,6 \%)$ & $12(48 \%)$ & $2(11,1 \%) *$ \\
\hline No conoce & $29(67,4 \%)$ & $13(52 \%)$ & $16(88,9 \%)$ \\
\hline \multicolumn{4}{|c|}{ Vacunas en las que debe aplicar «test agitación» } \\
\hline Adsorbidas & $3(7,0 \%)$ & $3(12 \%)$ & - \\
\hline Bacterianas & $10(23,3 \%)$ & $7(28 \%)$ & $3(16,7 \%)$ \\
\hline Víricas & $1(2,3 \%)$ & $1(4 \%)$ & - \\
\hline No conoce & $29(67,4 \%)$ & $14(56 \%)$ & $15(83,3 \%)$ \\
\hline \multicolumn{4}{|c|}{ Congelación afecta vacuna frente DTP } \\
\hline No & $18(41,9 \%)$ & $9(36 \%)$ & $9(50,0 \%)$ \\
\hline Sí & $23(53,5 \%)$ & $15(60 \%)$ & $8(44,4 \%)$ \\
\hline Desconoce & $2(4,7 \%)$ & $1(4 \%)$ & $1(5,6 \%)$ \\
\hline \multicolumn{4}{|c|}{ Congelación afecta vacuna frente VHB } \\
\hline No & $19(44,2 \%)$ & $9(36 \%)$ & $10(55,6 \%)$ \\
\hline Sí & $22(51,2 \%)$ & $15(60 \%)$ & $7(38,9 \%)$ \\
\hline Desconoce & $2(4,7 \%)$ & $1(4 \%)$ & $1(5,6 \%)$ \\
\hline \multicolumn{4}{|c|}{ Congelación afecta vacuna frente VPO } \\
\hline No & $19(44,2 \%)$ & $14(56 \%)$ & $25(27,8 \%)$ \\
\hline Sí & $22(51,2 \%)$ & $10(40 \%)$ & $12(66,7 \%)$ \\
\hline Desconoce & $2(4,7 \%)$ & $1(4 \%)$ & $1(5,6 \%)$ \\
\hline \multicolumn{4}{|c|}{ Congelación afecta vacuna frente RSP } \\
\hline No & $23(53,5 \%)$ & $12(48 \%)$ & $11(61,1 \%)$ \\
\hline Sí & $18(41,9 \%)$ & $12(48 \%)$ & $6(33,3 \%)$ \\
\hline Desconoce & $2(4,7 \%)$ & $1(4 \%)$ & $1(5,6 \%)$ \\
\hline
\end{tabular}

\section{DISCUSIÓN}

La tasa de participación en nuestro estudio fue del $93,5 \%$, que puede considerarse como excelente, al mejorar incluso los valores publicados por otros autores en países desarrollados $7,17,18$.

El objetivo principal de este trabajo de investigación era evaluar como se realizaba el mantenimiento de la cadena del frío en distintos puntos de vacunación en atención primaria de salud, ya que como hemos comentado, es en este ámbito donde fundamental- mente se lleva a cabo e indirectamente, el buen funcionamiento de los programas de inmunización. No hay duda de que en primer lugar es esencial valorar la figura del responsable de las vacunas. Este profesional debe conocer las características de termoestabilidad de dichos preparados, con el fin de evitar errores durante su manipulación, y en caso de producirse ser capaz de detectarlos y subsanarlos.

A este respecto, en nuestro trabajo el $93 \%$ de los puntos de vacunación visitados tenían un único responsable y en el resto no más de 
dos. Es indudable la importancia que puede tener este hecho en la correcta conservación de estos preparados, máxime cuando estudios semejantes, como los de Bishai $^{17}$ y Liddle $^{19}$, ya ponían de manifiesto que solo en un $20-38 \%$ de los casos se podía identificar a un responsable único en los centros encuestados. En otros estudios ${ }^{3}$, hasta un 5\% de los casos no existía un responsable reconocido y recientemente Hazelton, en $2001^{20}$,comprobó cómo en más del $6 \%$ de los puntos de vacunación carecían de responsable, demostrando que este déficit está fuertemente asociado con errores durante el mantenimiento de las vacunas.

En nuestra opinión, que exista un único responsable y que éste sea titulado sanitario, avalaría a priori una adecuada formación y profesionalidad de los encuestados para hacerse cargo del control y mantenimiento de los preparados vacunales. Por otro lado, hemos de resaltar que todos los encuestados estaban muy sensibilizados sobre la importancia de la correcta conservación de estos preparados y su papel para que esto sea así.

No obstante, hemos detectado algunas deficiencias en ciertos aspectos que podrían incidir en la efectividad de los programas de vacunación. En primer lugar la ubicación incorrecta de las vacunas en el interior de los frigoríficos, ya que en un $14 \%$ de las ocasiones las observamos situadas en la puerta de los mismos; esta cifra prácticamente duplica $(8 \%)$ a la reseñada por De Campo en un estudio efectuado en Australia ${ }^{22}$.

En segundo lugar, en tres ocasiones detectamos desviaciones en la temperatura que marcaba el termómetro en el momento de la visita (en 2 era inferior a $0^{\circ} \mathrm{C}$; en uno de los casos era una situación reiterada que se había puesto en conocimiento de los superiores, y en otro de los puntos de vacunación el frigorífico marcaba $8,5^{\circ} \mathrm{C}$ ).

En tercer lugar, en relación con la información que el responsable poseía con respecto a la termoestabilidad de las vacunas, la totalidad de los profesionales tenían muy claro que la vacuna de polio oral es la más sensible a los cambios de temperatura, lo que revela la eficacia de la información que han venido recibiendo en este sentido. Pero por el contrario hemos podido constatar que sería recomendable incidir sobre la inestabilidad que presentan las vacunas adsorbidas cuando se someten a congelación, ya que casi un 50\% consideraba que las bajas temperaturas no afectaban a estos preparados.

Otro punto a destacar es el relativo al desconocimiento de los encuestados sobre el denominado «test de agitación», ya que sólo el 32\% de los mismos afirmó conocerlo, a pesar de tratarse de un procedimiento sencillo y de fácil aplicación, que alerta inmediatamente sobre la posibilidad de que un determinado vial se haya inactivado por exposición a bajas temperaturas ${ }^{4,14}$.

En general, los resultados de nuestro trabajo manifiestan una alto cumplimiento de las recomendaciones para el almacenamiento de las vacunas, a diferencia de lo observado en otros estudios nacionales e internacionales ${ }^{3,9,26}$, donde las normativas nacionales eran cumplidas por un escaso porcentaje de profesionales.

Además, en lo que se refiere a nuestro país, estudios anteriores, como el realizado por Fernando Valls ${ }^{27}$, revelaban graves deficiencias, incluso la inexistencia de termómetros en el interior del frigorífico, lo que impedía valorar las condiciones en que se encontraban las vacunas. Un estudio posterior $^{28}$ demostraba que sólo el $80 \%$ de los frigoríficos tenían termómetro interior y sólo en 7,69\% de los casos se realizaba un control diario correcto de la temperatura. Estas carencias coincidían claramente también con los resultados de otros estudios similares realizados en países desarrolla$\operatorname{dos}^{3,6,16,18,22}$.

Por otra parte, en los 43 puntos de vacunación visitados en nuestro trabajo, el frigorífico se destinaba exclusivamente a la conser- 
vación de las vacunas y medicamentos termosensibles. En ningún caso se visualizó el almacenamiento conjunto de alimentos o bebidas ni otros productos químicos. Esto contrasta con lo apreciado por otros autores ${ }^{16}$ quienes detectaban la presencia de estos productos hasta en el $62 \%$ de los aparatos visitados. Evidentemente esto podría explicar las escasas desviaciones $(6,97 \%)$ del rango óptimo de temperatura detectadas por nosotros, especialmente si las comparamos con los datos de otros autores que registran lecturas anómalas hasta en el $75 \%$ de los frigoríficos monitorizados $^{3}$. Estos datos cobran aún mayor relevancia si tenemos presente que los recursos materiales de los que se disponía presentaban importantes carencias, como es el hecho de que menos del $40 \%$ de los frigoríficos eran modelos específicamente sanitarios, sólo en tres ocasiones era posible visualizar la temperatura sin necesidad de abrir el frigorífico y tan sólo en un punto de vacunación el frigorífico estaba conectado a una red eléctrica de emergencia.

Por todo ello, creemos necesario recomendar el reforzamiento de la información y formación de los responsables de vacunas sobre la inestabilidad de estos preparados, sobre todo frente a la congelación. Y por supuesto adecuar al progresivo aumento de la importancia de los programas de inmunización los recursos materiales que no se han adaptado convenientemente a las nuevas necesidades.

\section{AGRADECIMIENTOS}

Queremos expresar nuestro agradecimiento tanto a la Dirección del Área Sanitaria núm 1 del Insalud por habernos brindado la oportunidad para realizar este estudio, como a todos los profesionales que han participado en él. Queremos resaltar que nos hemos encontrado con un grupo de profesionales conscientes de su responsabilidad en el mantenimiento de la calidad de los programas de inmunización y extraordinariamente sensibilizados por la trascendencia que tiene la cadena del frío para garantizar la efectividad de los mismos.

\section{BIBLIOGRAFÍA}

1. Scholtz M, Duclos P. Immunization safety: a global priority. Bull World Health Org 2000; 78: 153-4.

2. Batalla J, Fernández-Lara N. Pautas de transporte, distribución y conservación de las vacunas. En: Salleras L1, editores. Vacunaciones Preventivas. Principios y aplicaciones. Barcelona: Masson, SA; 1998. p.543:63.

3. Thakker Y, Woods S. Storage of vaccines in the community: weak link in the cold chain? BMJ 1992;304:756-8.

4. WHO/EPI/LHIS. Safe vaccine handling, cold chain and immunizations. A manual for the Newly Independent States. Documents WHO/EPI/LHIS/98.02. Geneva: OMS; 1998.

5. Watson JC, Peter G. General Inmunization Practices. In: Plotkin SA, Orenstein WA, editores. Vaccines. 3 ed. Philadelphia: WB Saunders Company; 1999. p.47-73.

6. Yuan L, Daniels S, Naus M, Brcic B. Vaccine storage and handling. Knowledge and practice in primary care physicians'offices. Can Fam Physician 1995; 41:1169-76.

7. Steinmetz N, Furesz J, Reinhold C, Yarosh W Storage conditions of live measles, mumps and rubella virus vaccines in Montreal. Can Med Assoc J 1983; 128:162-3.

8. Chen RT, Hardy IR, Rhodes PH, Tyshchenko DK, Moiseeva AV, Marievsky VF. Ukraine, 1992: First Assessment of Diphtheria Vaccine Effectiveness during the Recent Resurgence of Diphtheria in the Former Soviet Union. J Infect Dis 2000; 181 Suppl 1: S178-83.

9. Gold MS, Kemp AE, Ousbourne M. Counting the cost of disrupting the vaccine cold chain. Med J Aust 1998; 168:471-2.

10. Centers for Disease Control and Prevention. US Department of Health and Human Services, $\mathrm{Pu}$ blic Health Service. Vaccine Management: Recomendations for Handling and Storage of Selected Biologicals. Atlanta: Centers for Disease Control and Prevention; 1999.

11. Diminsky D, Moav N, Gorecki M, Barenholz Y Physical, chemical and immunological stability of CHO-derived hepatitis B surface antigen (HBsAg) particles. Vaccine 2000; 18: 3-17.

Rev Esp Salud Pública 2002, Vol. 76, N. ${ }^{\circ} 4$ 
12. Otto BF, Suarnawa M, Stewart T, Nelson C, Ruff TA, Widjaya A, Maynard JE. At-birth immunisation against hepatitis B using a novel pre-filled immunisation device stored outside the cold chain. Vaccine 2000; 18: 498-502.

13. Centers for Disease Control and Prevention. US Department of Health and Human Services, Public Health Service. Guidelines for Vaccine Packing and Shipping. Atlanta: Centers for Disease Control and Prevention; 1997.

14. WHO/V\&B. Product information sheets 2000 . Documents WHO/V\&B/00.13. Geneva: OMS; 2000.

15. WHO/EPI/LHIS. Temperature monitors for vaccines and the cold chain. Documents WHO/EPI/ LHIS/99.15. Geneva: OMS; 1999.

16. Grasso M, Ripabelli G, Sammarco ML, Manfredi TM, Quaranta A. Vaccine storage in the community: a study in central Italy. Bull World Health Org 1999; 77:352-5.

17. Bishai DM, Bhatt S, Miller LT, Hayden GF. Vaccine Storage Practices in Pediatric Offices. Pediatrics 1992;89:193-6.

18. Jeremijenko A, Kelly H, Sibthorpe B, Attewell R. Improving vaccine storage in general practice refrigerators. BMJ 1996;313:1651-2.

19. Liddle J, Harris M. How general practitioners store vaccines. A survey in south-western Sydney. Med J Aust 1995;162:366-8.

20. Hazelton DJ, Bowd K, Liddle J, Balcomb A, Hazelton KA. Med J Aust 2001;174:545.
21. Finn L, Crook S. A district survey of vaccine cold chain protection in general practitioners' surgeries. Commun Dis Public Health 1999; 2(1): 47-9.

22. de Campo MO, Lester R. Maintenace of the vaccine cold chain by councils and general practices in Victoria. Med J Aust 1998; 168: 365-6.

23. Consejería de Salud. Dirección General de Prevención y Promoción de la Salud. Comunidad de Madrid. Manual de Inmunizaciones. Documento Técnico de Salud Pública 1994;14:91-6.

24. Hulley SB, Cummings SR. Planning the Measurements: Precision and Accuracy. In: Designing Clinical Research. An Epidemiologic Approach. Williams \& Wilkins. Baltimore 1988.

25. Norusis MJ. SPSS. Base System para Windows Versión 10.0. Licencia n. ${ }^{\circ} 301012956368110$ 33563855221063.

26. Gylca R, Gylca V, Benes O, Melnic A, Chicu V, Weisbecker C, Willems P, Kaufhold A. A new DTPa-HBV-IPV vaccine co-administered with Hib, compared to a commercially available DTPw-IPV/Hib vaccine co-administered with $\mathrm{HBV}$, given at 6,10 and 14 weeks following HBV at birth. Vaccine 2001; 19: 823-33.

27. Fernando Valls T, Guevara Sarrano J, Haro Salinas M, Llopis González A. Estado actual de la cadena de frío de vacunas en las Comunidades Autónomas de Aragón y La Rioja. Pharmaklinik 1989; 3:18-23.

28. Fernando Valls T, Tuells Hernández J, Llopis González A, Guevara Sarrano J, Haro Salinas M, Villalba Garnica P, Morales Suárez-Varela M. Cadena de frío de vacunas. Estudio del material inmunizante para campañas de vacunación en España. Farm Clin 1989;6:212-20. 
ANEXO 1

Protocolo para evaluar la cadena del frío en los equipos de atención primaria

\begin{tabular}{|l|l|}
\hline N. ${ }^{\text {Entrevista: }}$ & Fecha: \\
\hline Sexo: & Edad: \\
\hline
\end{tabular}

\section{Frigorífico}

1. El frigorífico es de uso:

$\square$ Exclusivo para vacunas

$\square$ Exclusivo para medicamentos termosensibles

$\square$ Otros usos. Especificar

2. El frigorífico se encuentra conectado a la red:

$\square$ Directamente

$\square$ Mediante derivaciones

3. ¿Disponen de Dispositivo de Alarma para detectar fallos en el suministro eléctrico? :

$\square$ Sí, Especificar

$\square$ No

4. ¿El frigorífico se encuentra conectado al circuito de emergencia del centro de manera que en caso de corte de suministro eléctrico continúe recibiendo corriente? :

$\square$ Sí

$\square$ No

5. El control de la Temperatura de su frigorífico se realiza mediante:

Termómetros de máxima y mínima

Termómetros convencionales

$\square$ Sin termómetro

6. Para realizar la lectura del termómetro, ¿es necesario abrir la nevera?:

Sí

$\square$ No 


\section{ANEXO 1 (continuación)}

\section{Protocolo para evaluar la cadena del frío en los equipos de atención primaria}

7. El control de la Temperatura se realiza:

$\square 2$ veces al día

$\square 1$ vez al día

$\square$ 1-2 veces a la semana

1 vez al mes

$\square$ No se realiza monitorización de la temperatura

8. ¿El frigorífico dispone de alarma en la puerta que impida que ésta quede abierta accidentalmente?:

$\square$ Sí

$\square$ No

9. ¿El frigorífico dispone de algún método para mantener la temperatura en el interior?:

$\square$ Botellas de agua o solución salina en el compartimento inferior

$\square$ Bolsas Ice-pack en el compartimento inferior

$\square$ Otros. Especificar

No requiere

10. Si el frigorífico necesita descongelación periódica, ¿qué procedimiento sigue?:

$\square$ Utilización de otro frigorífico

$\square$ Utilización de neveras portátiles con acumuladores

$\square$ No requiere descongelación

11. En caso necesario dispone de congelador para mantener las bolsas Ice-park:

$\square$ Sí

$\square$ No

12. Temperatura del frigorífico cuando se realiza la visita:

13. Las vacunas se encuentran situadas en:

$\square$ Bandejas centrales, separadas de las paredes 


\section{ANEXO 1 (continuación)}

Protocolo para evaluar la cadena del frío en los equipos de atención primaria

$\square$ En la puerta

$\square$ Otras localizaciones

14. La ubicación de las vacunas en el frigorífico se realiza en función de:

$\square$ Nivel de ocupación

Termoestabilidad de la vacuna

La fecha de caducidad de la vacuna

$\square$ Termoestabilidad de la vacuna y fecha de caducidad

15. Presencia de alimentos / bebidas en frigorífico:

$\square$ Sí

$\square$ No

16. Presencia de alimentos bebidas en congelador:

$\square$ Sí

$\square$ No

17. Presencia de vacunas caducadas:

$\square$ Sí

$\square$ No

18. ¿Se encuentran distintos viales abiertos de una misma vacuna en el frigorífico?

$\square$ Sí

$\square$ No

19. El responsable de la cadena del frío es:

$\square$ Una persona concreta

$\square$ Más de una persona

20. La titulación del responsable es:

Medicina

$\square$ ATS/DUE 


\section{ANEXO 1 (continuación)}

\section{Protocolo para evaluar la cadena del frío en los equipos de atención primaria}

$\square$ Auxiliar

$\square$ Otros. Especificar

21. ¿Se registra la Temperatura diariamente en las gráficas mensuales? :

$\square$ Sí

$\square$ No

22. ¿Durante el último año ha sufrido algún tipo de avería el frigorífico?

Nunca

$\square$ Una vez

Más de una vez

23. ¿Conoce Ud. las Normas de Actuación recomendadas ante un problema en la conservación de las vacunas?

$\square$ Sí

$\square$ No

24. ¿Qué rango de Temperatura considera óptimo para la conservación adecuada de la mayoría de las vacunas?

$\square$ 2. ${ }^{\circ}-8 .^{\circ} \mathrm{C}$

$\square 0 .^{\circ}-8 .^{\circ} \mathrm{C}$

2. ${ }^{\circ}-10 .^{\circ} \mathrm{C}$

25. Las vacunas pierden actividad por la exposición:

$\square$ A altas Temperaturas

A altas y bajas Temperaturas

$\square$ No se alteran con los cambios de Temperatura

26. ¿Conoce el test de agitación y para que se aplica?

$\square$ Sí

$\square$ No

Rev Esp Salud Pública 2002, Vol. 76, N. ${ }^{\circ}$ 


\section{ANEXO 1 (continuación)}

Protocolo para evaluar la cadena del frío en los equipos de atención primaria

27. ¿Podría decirme cuál/es de las siguientes vacunas ven modificada su eficacia cuando se almacenan a Temperatura inferior a $0^{\circ} \mathrm{C}$ ?

Vacuna antitetánica

$\square$ Vacuna antihepatitis B

$\square$ Vacuna de polio oral

$\square$ Vacuna triple vírica (RSP)

28. Durante la jornada diaria de vacunación, los viales de las diferentes vacunas que se aplican se conservan:

$\square$ En el frigorífico

Sobre la mesa de trabajo, para agilizar la administración de la vacuna

29. ¿Con qué periodicidad se realiza el pedido de vacunas fuera de campañas vacunales?:

$\square$ Mensual

$\square$ Bimensual

$\square$ A demanda

$\square$ Otros, especificar.

30. Cuando se recibe el pedido de vacunas se realiza alguna de las siguiente comprobaciones:

Comprobación tipo de vacunas y número de dosis recibidas se corresponden con los solicitados

$\square$ Comprobación del estado y etiquetaje de los viales de las vacunas.

$\square$ Verificación de los indicadores de tiempo y temperatura.

Se realizan las tres comprobaciones anteriormente citadas.

$\square$ No se realiza ninguna comprobación. 